Published in final edited form as:

Clin Obstet Gynecol. 2012 June ; 55(2): 550-559. doi:10.1097/GRF.0b013e31824df256.

\title{
Measles, Mumps, and Rubella
}

\author{
Sarah J. White, Ph.D. ${ }^{1,2}$, Kristi L. Boldt, M.D. ${ }^{3}$, Sara J. Holditch, B.S. ${ }^{4}$, Gregory A. Poland, \\ M.D. ${ }^{1,2}$, and Robert M. Jacobson, M.D. ${ }^{1,5}$ \\ ${ }^{1}$ Mayo Clinic Vaccine Research Group, Mayo Clinic 200 First Street SW Rochester MN, 55905 \\ ${ }^{2}$ Program in Translational Immunovirology and Biodefense, Mayo Clinic 200 First Street SW \\ Rochester MN, 55905
}

${ }^{3}$ Department of Obstetrics and Gynecology, Mayo Clinic 200 First Street SW Rochester MN, 55905

${ }^{4}$ Mayo Graduate School, Mayo Clinic 200 First Street SW Rochester MN, 55905

${ }^{5}$ Department of Pediatric and Adolescent Medicine, Mayo Clinic 200 First Street SW Rochester MN, 55905

\begin{abstract}
Measles, mumps, and rubella are viral diseases that may adversely affect non-immune pregnant women and their fetuses/neonates. Prevention of these diseases and their complications can be achieved through measles-mumps-rubella (MMR) vaccination prior to pregnancy. The vaccine is contraindicated during pregnancy because it contains live, attenuated viruses that pose a theoretical risk to the fetus. However, accidental receipt of MMR vaccination is not known to cause maternal/fetal complications. MMR immunization is recommended to non-immune obstetric patients upon completion or termination of pregnancy.
\end{abstract}

\section{Keywords}

Measles-Mumps-Rubella Vaccine; Measles; Mumps; Rubella; Congenital Rubella Syndrome; Obstetrics

\section{Introduction}

Measles, mumps, and rubella are vaccine-preventable, viral diseases that have the potential to harm non-immune pregnant women and their fetuses/neonates if exposure occurs. ${ }^{1-9}$ The most concerning of these is rubella as it can cause congenital rubella syndrome (CRS) with devastating effects. ${ }^{3,4,6,7}$ In this review, we consider each of the diseases in turn and address the role of vaccination in preventing these diseases and their complications.

\footnotetext{
Corresponding Author: Robert M. Jacobson, M.D. Mayo Clinic Desk Ba3b 200 First St SW Rochester, MN 55902-0001 Jacobson.Robert@mayo.edu Telephone number: (507)538-1642 Fax number: (507)284-9744.

Conflict of Interest

Dr. Poland chairs data monitoring committees for non-measles vaccines being developed by Merck \& Co. Dr. Jacobson also serves on a data monitoring committee as well as a safety review committee concerning non-measles vaccines produced by Merck \& Co. For the remaining authors, no conflicts of interest were declared.

Publisher's Disclaimer: This is a PDF file of an unedited manuscript that has been accepted for publication. As a service to our customers we are providing this early version of the manuscript. The manuscript will undergo copyediting, typesetting, and review of the resulting proof before it is published in its final citable form. Please note that during the production process errors may be discovered which could affect the content, and all legal disclaimers that apply to the journal pertain.
} 


\section{Measles}

\section{Etiology}

Measles, or rubeola, is an RNA virus, belonging to the Morbillivirus genus, which is in the Paramyxoviridae family. ${ }^{1,5}$ Humans are the only natural host of this highly contagious virus.

\section{Epidemiology}

Measles is transmitted through droplet nuclei. In temperate regions, the incidence is highest in late winter and spring ${ }^{1,4,5}$ Reports of measles cases in the U.S. have dramatically declined since the pre-vaccine era. In 2000, the Centers for Disease Control and Prevention (CDC) declared that measles was eliminated from the U.S., although outbreaks resulting from foreign travel still occur. ${ }^{10}$ From January to September 2011, 15 measles outbreaks with 211 confirmed cases were reported in the U.S., the highest number since 1996. Out of the $211,18 \%$ occurred among individuals who received at least one MMR vaccine dose. Until measles is eradicated, outbreaks will continue in the U.S. and worldwide. Currently, over 20 million measles infections occur worldwide annually, with 164,000 deaths in 2008 alone. $^{1}$

\section{Populations susceptible to exposure}

The risk of exposure is higher for certain populations. ${ }^{1,4}$ For instance, epidemics still occur, typically in developing countries without mass vaccination programs. Close contact with non-vaccinated individuals from these countries (e.g., airports, clinics, and hospitals) increases the chance of exposure among non-immune individuals.

Measles is thought of as a childhood disease, but demographics have shifted. ${ }^{1,4}$ Since 2001, half of the reported cases in the U.S. were in those 20 years and older. Although outbreaks are rare in the U.S., an individual case could lead to an outbreak due to the high transmissibility of the virus. Obstetrical care providers should be aware of any reported measles cases in the area and, if so, monitor non-vaccinated obstetric patients closely for both exposure to measles and its clinical manifestations.

\section{Clinical manifestations}

Measles transmission occurs by droplet nuclei. ${ }^{1,4,5,8}$ Communicability lasts approximately eight days. The prodromal stage occurs 10 to 12 days after exposure and is characterized by two to three days of fever, anorexia, and malaise combined with the triad of cough, conjunctivitis, and coryza. ${ }^{1,4,5}$ Towards the end of the prodromal stage, Koplik's spots, an enanthem comprised of blue-white spots, appear on the buccal surfaces of the mouth and last 12 to 18 hours. ${ }^{1,5,8}$ They are pathognomonic of measles infection. ${ }^{1,5}$ The prodromal phase is followed by the appearance of a maculopapular, erythematous rash, accompanied by a high fever. The rash occurs anywhere from one or two days before to one or two days after the Koplik's spots appear, lasting five to six days in toto. ${ }^{1}$ The rash begins (and disappears) on the head and face, expanding outwards and downwards, eventually reaching the hands and feet. A persisting cough characterizes the convalescent stage, which may persist up to one to two weeks after the rash resolves. ${ }^{5}$

Measles-induced complications affect approximately $30 \%$ of infected individuals, especially young children (ages < 5) and adults (ages $\geq 20$ ). ${ }^{1,4}$ The most commonly reported complications are diarrhea (8\%), otitis media (7\%), and pneumonia (6\%). ${ }^{1,4,5,8}$ The leading cause of death in adults is acute encephalitis, a rare complication of measles $(0.1 \%){ }^{1,4}$ Historical data suggest that complications are more severe in pregnant women. ${ }^{5}$ 


\section{Complications in the obstetric patient and her offspring due to infection}

Measles exposure during pregnancy may cause adverse maternal and fetal effects. ${ }^{1,4,5,8,9}$ In a CDC study, 58 pregnant women with active measles infection were followed to assess measles-induced maternal and fetal effects. ${ }^{9}$ Fifteen of the 58 women developed pneumonia, of which two died. The most common fetal/neonatal effect observed was premature delivery (13 of 58). In addition, five pregnancies resulted in spontaneous abortion. Measles has not been proven to cause birth defects. ${ }^{1,4,5,8}$

If a non-immune pregnant patient is exposed to measles just before delivery, in utero and intrapartum viral transmission is likely to cause a serious infection in the neonate. ${ }^{5}$ The risk can be reduced with passive immunization (see post-exposure interventions).

\section{Diagnosis of infection}

One confirms diagnosis with a seropositive antibody response using a serological assay as well as detection of measles in clinical specimens (e.g., urine, nasopharyngeal secretions, throat swabs, or blood) by viral culture. ${ }^{1,4,5}$ Blood samples for serological assays should be drawn at the same time as the collection of clinical specimens; however, clinical specimens should be collected no later than seven to ten days after the rash onset. ${ }^{1}$ Serological assays (e.g., enzyme-linked immunosorbent assays [ELISA], hemagglutination inhibition assays [HIA]) that measure IgM titers can be performed in a medical laboratory, although the most reliable serological assay is an IgM capture assay developed and performed by the CDC. ${ }^{1,4}$ The IgM capture assay can only be performed within 72 hours after rash onset, whereas serological tests performed in a medical laboratory at a hospital or medical facility can detect IgM one to two months after the onset of symptoms. Due to the high transmissibility of measles, virus isolation of clinical specimens from individuals suspected of measles exposure is performed by the state public health laboratory or the CDC. ${ }^{1}$

\section{Post-exposure interventions}

Immune globulin is a recommended prophylaxis for non-immune, exposed obstetric patients as long as treatment is received no more than six days after exposure. ${ }^{1,4,5}$ Immune globulin is administered intramuscularly at $0.25 \mathrm{ml} / \mathrm{kg}$ (maximum dose $=15 \mathrm{ml}$; given at two injection sites). It will not prevent measles but may suppress the measles-induced symptoms until the MMR vaccination can be administered upon completion or termination of pregnancy. Additionally, if the maternal infection is close to parturition, immune globulin may reduce the risk of the neonate developing measles. Otherwise, treatment for measles in the obstetric patient is limited to symptomatic care. ${ }^{5}$

\section{Mumps \\ Etiology}

Mumps, like measles, is a disease caused by an RNA virus classified in the Paramyxoviridae family. ${ }^{2,5,8}$ Unlike measles, mumps belongs to the Rubulavirus genus. It is a human disease with no animal reservoirs.

\section{Epidemiology}

Mumps cases can occur at any time of the year with peak incidences during winter and spring. ${ }^{2,5}$ While reports of mumps cases in the U.S. have declined by more than $90 \%$ since the pre-vaccine era (212,000 reported cases in 1968), mumps outbreaks still occur in the U.S. and worldwide. The largest mumps outbreak in decades occurred through the Midwest in 2006 (6,584 cases) followed by outbreaks along the East Coast and Canada in 2009-2010 
(3,502 cases). Among those for whom records were available in the 2009-2010 outbreak, $90 \%$ had received at least one dose of mumps-containing vaccine. ${ }^{2,11}$

\section{Populations susceptible to exposure}

Mumps viral transmission occurs through contact with droplets of saliva or mucus from the mouth, nose, or throat of an infected person, anywhere from three days before to four days after the onset of parotitis, the disease's hallmark characteristic. ${ }^{2,4}$ However, infected individuals lacking classical symptoms also have the potential to transmit mumps to people susceptible to exposure.

While individuals from countries without routine immunization against mumps are more likely to import mumps to the U.S., internationally-imported mumps cases in the U.S. are also possible from countries with routine mumps immunization programs. ${ }^{2,4,11}$ For instance, an 11 year-old boy with mumps traveling from the United Kingdom in 2009 transmitted the disease to people susceptible to exposure in New York, eventually spreading across the East Coast and into Canada. ${ }^{1,11}$ Over half (53\%) of the reported cases from this multi-state outbreak involved school-aged children (ages 5-17). This is likely due to their close and consistent contact at school. Students exempted from receiving the MMR vaccine because of medical, religious, or philosophical reasons or because their parents fear the MMR vaccine causes autism are at a higher risk for exposure to mumps. Mumps transmission is also promoted by other areas where people may congregate, including airports, urban areas, colleges, and large households. $1,4,5$

\section{Clinical manifestations}

A prodromal stage appears 14 to 18 days after exposure and occurs before parotitis develops. This prodromal stage includes nonspecific symptoms of malaise, myalgia, headache, lowgrade fever, and anorexia. ${ }^{2}$ As many as $40-50 \%$ of the infected population have only nonspecific symptoms. Another $20 \%$ are asymptomatic. The remaining infected population is at risk for developing parotitis, which can be unilateral or bilateral. Salivary gland inflammation decreases after one week with full recovery by ten days. ${ }^{2}$ Complications include aseptic meningitis ( $₫ 5 \%)$, pancreatitis $(2-5 \%)$, oophoritis $(5 \%)$, meningoencephalitis (1/50,000 cases), and deafness (1/20,000 cases). ${ }^{1}$

\section{Complications in the obstetric patient and her offspring due to infection}

Unlike measles, pregnancy does not appear to affect mumps' severity, ${ }^{5}$ but if exposure occurs during the first trimester, the patient has a higher risk of spontaneous abortion. ${ }^{4,5,8}$ Spontaneous abortion has been found to occur in $27 \%$ of pregnant women exposed to mumps during their first trimester compared to $13 \%$ of controls. ${ }^{8}$ There are no associated congenital malformations in children whose mothers were exposed to mumps at any time during pregnancy. $2,4,5,11$

\section{Diagnosis of infection}

A laboratory diagnosis of mumps may be needed if exposure to mumps is suspected. Mumps can be diagnosed by isolating the virus from a cultured swab of one of the inflamed salivary glands, particularly the parotid duct. ${ }^{2,4,5}$ Mumps may also be diagnosed from a buccal or throat swab using polymerase chain reaction (PCR). ${ }^{2,4} \mathrm{~A}$ commonly used, simple, and sensitive means to diagnose mumps infection is by measuring $\operatorname{IgM}$ or IgG titers using a serological test (e.g., ELISA). ${ }^{2,5}$ IgM titers are useful during the initial period of the illness but are often not detectable in individuals with one or more doses of mumps-containing vaccine. IgG titers are useful from a time between the exanthema and the recovery phase of infection to years after infection. False negatives are possible and, therefore, diagnosis 
should not be necessarily dismissed if the exposure has not been laboratory-confirmed, but parotitis and other common mumps manifestations are observed.

\section{Post-exposure interventions}

Treatment for mumps infection is limited to symptomatic relief. ${ }^{5}$

\section{Rubella \\ Etiology}

Rubella, or German measles, is an RNA virus in the genus Rubivirus within the Togaviridae family. ${ }^{3,6}$ It is a human disease with no animal reservoirs.

\section{Epidemiology}

The incidence of rubella cases is highest from late winter to early spring. ${ }^{3}$ The number of rubella cases has progressively declined in the U.S. since the pre-vaccine era due to mass immunization programs and, as of 2004, is no longer endemic. ${ }^{3}$ Rubella remains endemic in other countries with a dramatic increase in cases reported during 2009 versus during $2000 .{ }^{12}$ The World Health Organization (WHO) reported that rubella cases in the African Region and the South-East Asian Region alone increased from 865 to 17,388 and from 1,165 to 17,208 during 2000 and 2009, respectively. These regions represent a significant number of the 121,344 global cases of rubella reported by the WHO during 2009; neither region has any specific goals to control rubella outbreaks. Rubella eradication will not be possible unless global immunization policies (e.g., immunization registries) are implemented and enforced.

\section{Populations susceptible to exposure}

The majority (71\% as of 2003) of rubella cases in the U.S. are currently among individuals of child-bearing ages (ages 15-39). ${ }^{3,4}$ This shift in demographics from a classical childhood disease increases the probability of Congenital Rubella Syndrome (CRS) development should a rubella outbreak occur. Rubella cases reported in the U.S. presently involve a high proportion of Hispanic-born young adults from countries lacking routine administration of rubella-containing vaccines. Consequently, the majority of CRS cases reported in the U.S. since 1997 are among infants and children born to foreign-born women from Latin American and Caribbean countries. ${ }^{3}$

Transmission occurs via contact with droplets from infected individuals during the last week of the two- to three-week incubation period, until a week post-development of the stereotypic rubella rash. ${ }^{3,6}$ Individuals with asymptomatic or subclinical infections may also transmit the disease. Additionally, infants with CRS shed large quantities of the rubella virus from their bodily secretions for more than a year post-parturition and are highly contagious. Non-immune pregnant women in close contact with infected individuals are at risk for acquired rubella infection and their offspring may develop rubella-induced complications.

\section{Clinical manifestations}

Acquired rubella infection may be asymptomatic or subclinical in up to half of those exposed, especially in children. ${ }^{3,4,6}$ Of the people who clinically manifest the disease, symptoms are mild and self-limiting. A prodromal stage of one to five days is represented by a low-grade fever, malaise, lymphadenopathy and an upper respiratory infection. Forchheimer spots (petechiae on the soft palate) may precede or accompany the rash. ${ }^{3,6}$ The rash is mild and maculopapular, beginning on the face and extending downwards; it occurs approximately 14 to 17 days after exposure and typically lasts three days. Rubella frequently 
leads to arthralgia/arthritis in women (up to 70\%). While joint symptoms, along with conjunctivitis, are more common complications in the obstetric patient, encephalitis $(1 / 6,000$ cases) may develop, affecting female adults more frequently than men or children. 3,4

\section{Complications in the obstetric patient and her offspring due to infection}

Although acquired rubella infection is generally mild, transplacental passage of the virus to the embryo/fetus during maternal viremia (five to seven days after exposure) can cause devastating consequences (i.e., CRS). ${ }^{3,4,6}$ Virus transmission with maternal re-infections are rare. ${ }^{6}$ CRS symptoms include, but are not limited to, fetal death, spontaneous abortion, premature delivery, ocular abnormalities (e.g., cataracts and microophthalmia), neurological problems (e.g., intellectual disability), abnormal cardiac development and, most commonly, deafness. Congenital malformations may be present at birth or sometimes develop months to years after birth. ${ }^{3,4,6}$ Examples of delayed CRS-induced maladies are type I diabetes mellitus, deafness, intellectual disability, subacute encephalitis.

The frequency and severity of CRS decreases as gestation progresses. ${ }^{3,4,6}$ For example, maternal exposure to rubella during the first 12 weeks of pregnancy results in CRS in $85 \%$ of developing embryos/fetuses. Conversely, by the $20^{\text {th }}$ gestational week, the risk of congenital defects is minimal. Nevertheless, neonatal rubella infections are possible when non-immune mothers transmit rubella to the fetus close to delivery. ${ }^{6}$

\section{Diagnosis of infection}

If exposure to rubella is suspected in a non-immune obstetric patient, laboratory diagnosis is necessary immediately, even if clinical manifestations are not present. ${ }^{3,4,6}$ Isolation of rubella with cultured throat or nasal specimens provides an accurate diagnosis of rubella, although it is a labor-intensive process. ${ }^{3,6} \mathrm{~A}$ serological assay (ELISA) can be easily and quickly performed to detect the presence rubella-specific antibodies. IgM is detectable within seven to 10 days following the initial exanthema. ${ }^{3}$ False-positives may occur if the patient has a positive $\mathrm{Rh}$ factor, is infected with parvovirus, or has a positive heterophile test for infectious mononucleosis. ${ }^{3,4,6}$ Seven to 21 days after the first ELISA, one should obtain an additional ELISA to confirm the diagnosis. Infection is confirmed by a positive IgM, a fourfold rise in $\operatorname{IgG}$, or a positive culture.

If the obstetric patient is positive for rubella infection, rubella may be diagnosed in the fetus by collecting either a specimen of amniotic fluid, cord blood, or placental tissue by amniocentesis, cordocentesis, or chorionic villus sampling (respectively) for use in laboratory assays including PCR, fluorescence, in situ hybridization, or ELISA (to quantify rubella-specific IgM). ${ }^{13}$ Obstetrical care providers can use the information gained from one of these methods to monitor the fetus closely for distress and the presence of any congenital defects associated with CRS. Ultrasound diagnosis alone on an infected fetus would be difficult due to the spectrum of abnormalities possible.

\section{Post-exposure interventions}

There is no specific pharmacological treatment for acquired rubella infection. $3,4,6$ Intramuscular administration of immune globulin ( $20 \mathrm{ml}$; given at two injection sites) within 72 hours of exposure may be administered to the obstetric patient to suppress, but not eliminate, symptoms. ${ }^{4,6}$ Infants with CRS have been born to women who received immune globulin therapy soon after exposure. Therefore, it is not a routine prophylaxis and should only be administered if the patient chooses not to terminate her pregnancy. Otherwise, treatment is limited to symptomatic relief. ${ }^{6}$ 


\section{Prevention with MMR Vaccination}

\section{Background}

The MMR (M-M-R-II®, Merck, Whitehouse Station, NJ) vaccine is recommended by the Advisory Committee on Immunization Practices (ACIP), the American Congress of Obstetricians and Gynecologists (ACOG), the American Academy of Pediatrics (AAP), and the American Academy of Family Physicians (AAFP) for routine use in the prevention of measles, mumps, and rubella. ${ }^{14,15}$ The combination is as effective as the formerly available monovalent forms: measles (ATTENUVAX®), mumps (MUMPSVAX®), and rubella (MERUVAX ${ }^{\circledR}$ ) vaccines (Merck). ${ }^{14-16}$ The trivalent vaccine reduces the number of injections from three to one in a two dose series, avoids unnecessary delays and the problems of spacing live, attenuated vaccines, and protects against all three diseases simultaneously. ${ }^{15,16}$ There is no medical reason to favor separate vaccination over the combination vaccine. These reasons, along with manufacturing constraints, prompted Merck to discontinue production of monovalent ATTENUVAX®, MUMPSVAX®, and MERUVAX® in 2008. ${ }^{15}$

The M-M-R-II ${ }^{\circledR}$ vaccine contains live, attenuated viruses for measles (Edmonston-Enders viral strain), mumps (Jeryl Lynn viral strain), and rubella (Wistar RA 23/7 viral strain). ${ }^{1-4,14}$ The MMRV vaccine (ProQuad $\left.{ }^{\circledR}\right)$, which includes a vaccine against the Varicella virus or chicken pox, was introduced in 2005, but is not recommended for individuals over 13 years of age. ${ }^{1-3}$

\section{Dosage and schedule}

The M-M-R II@ vaccine is supplied as a single-dose vial. ${ }^{4,14}$ It must be kept cool and out of light, because of the live, attenuated viruses' instability to heat and light. ${ }^{1-4,14,16,17}$ When reconstituted, it must be used within eight hours or discarded. Upon reconstitution, $0.5 \mathrm{ml}$ of the vaccine is administered subcutaneously, usually in the outer region of the upper arm. ${ }^{4,14,17}$ The ACIP recommends an immunization schedule to maximize the time of protection against measles, mumps, and rubella infection by minimizing the time between interference of maternal antibodies and receipt of MMR vaccine. ${ }^{16}$ While the current recommended schedule is to receive the first dose at 12-15 months followed by the second dose at 4-6 years (before elementary school enrollment), vaccine status should be assessed at every medical encounter and individuals due for a dose of MMR vaccine should receive the vaccine at that time. ${ }^{1-6,14,17}$ Receipt of the two-dose MMR series is strongly advised for eligible, non-pregnant women at least 28 days apart with completion no less than one month before conception. ${ }^{1-7,18}$ Counseling is recommended to discuss both the importance of not becoming pregnant for at least 28 days following the MMR vaccine series because of the theoretical risk posed by the live albeit attenuated vaccine viruses. ${ }^{4,6,18}$

\section{Accidental MMR receipt during pregnancy}

The possibility of transmitting a live, attenuated virus to the fetus during pregnancy and the concomitant consequences of that virus on the developing fetus is a theoretical (but never demonstrated) concern in vaccinating pregnant or soon-to-be pregnant women with the MMR vaccine. ${ }^{1-7,16,18}$ The theoretical risk for fetal complications arising from MMR vaccination is $1.6 \% .^{3,6}$ From 1971 to 1989 , the CDC maintained the Vaccine in Pregnancy Registry to document women who were vaccinated before or during pregnancy and to monitor any complications. ${ }^{3,4,6}$ The registry consisted of 321 pregnant women (full-term). Among these women, there was no evidence of MMR-induced congenital defects. With the absence of observed risk, the CDC does not recommend termination of pregnancy if inadvertent vaccination occurs. ${ }^{1-4,6,7}$ However, individual counseling is recommended to educate women of the real- but low-theoretical risk. $3,7,18$ 


\section{Evidence of immunity}

The standard of care in the U.S. is to ascertain the seroconversion rate of rubella, although not measles or mumps, during early prenatal care via serological assays. ${ }^{4,6,7}$ If the patient is found to be non-immune to rubella, the MMR vaccine is recommended upon completion or termination of pregnancy and before discharge from the medical facility. ${ }^{3,4,6}$ Breastfeeding is not a contraindication to MMR vaccination. ${ }^{1-4,16}$ Postpartum MMR vaccination while breastfeeding will not alter the infant's immunization schedule.

If the MMR vaccine was not administered before pregnancy and/or the pregnant woman was seronegative for rubella antibodies, then potential exposure may be minimized for the pregnant woman by washing hands frequently with soap and water, minimizing close contact with those who are ill with viral-like conditions, and avoiding travel to countries where rubella is still endemic. In addition, household contacts should receive the MMR vaccine to provide a measure of protection for mother and unborn child until she is able to be vaccinated. ${ }^{3}$

\section{Reactogenicity}

The MMR vaccine is safe and well tolerated. ${ }^{1-4,14}$ Common adverse effects are typically mild and include a fever ( $\left.\geq 103^{\circ} \mathrm{F} ; 5-15 \%\right)$, which begins five to 12 days after vaccination and lasts several days; arthritis (10\%); arthralgia (25\%); and a rash (5\%). ${ }^{1-6,17}$

Thrombocytopenia ( $<1 / 30,000$ doses $)$ and encephalitis/encephalopathy $(1 / 1,000,000$ doses $)$ are severe adverse effects, but are very rare. ${ }^{1-3,7,16-18}$ As mentioned, the MMR vaccine is contraindicated in pregnant women and women trying to conceive. ${ }^{1-7,16}$ The MMR vaccine is also contraindicated in those with a history of a severe allergy to vaccine additives (neomycin and gelatin); previous hypersensitivity reaction to MMR/MMRV vaccine; moderate or severe acute febrile illness; altered immunocompetence (immune deficiency diseases, leukemia, lymphoma, generalized malignancy, or receiving therapy with corticosteroids, alkylating drugs, antimetabolites, or radiation); receipt of antibodycontaining blood products (IG, IVIG, specific IG) $\leq$ two weeks before vaccine or $\leq$ three months after vaccine; and a personal history of seizures of any etiology. ${ }^{1-4,14,15,17,18}$ Exceptions to patients with altered immunocompetence: HIV patients (dependent on level of immunosuppression); patients with leukemia remission and no chemotherapy for $\geq 3$ months; steroid use is $<2$ weeks, in a low to moderate dose, as a replacement therapy, or administered via topical, intraarticular, bursal, or tendon injection. ${ }^{14,16,18}$

\section{Immunogenicity and efficacy}

The MMR vaccine is immunogenic and effective against measles, mumps, and rubella infections and offers long-term immunity for many people who receive two recommended doses. ${ }^{1-6}$ Vaccine failure, either primary (failure to seroconvert) or secondary (waning immunity), is chiefly responsible for the susceptibility to measles, mumps, and/or rubella exposure despite MMR vaccination. Issues regarding primary vaccine failure have been largely addressed by implementing the two MMR dose schedule ${ }^{1-4}$ In fact, the measlescomponent of the MMR vaccine is effective in $95-98 \%$ of individuals after one dose and $>99 \%$ after two doses. ${ }^{1,4,5}$ Vaccine efficacy against rubella is similar to measles with a $>95 \%$ seroconversion rate after one dose and $>99 \%$ after two doses. $3,4,6$ The mumps component of the MMR vaccine is the least effective of the three live, attenuated viruses with $64-95 \%$ seroconversion after one dose and $88-95 \%$ seroconversion after two doses. ${ }^{2,4}$ In infants, the timing of the first MMR dose may also affect efficacy against the measlescomponent of the MMR vaccine if administered 12-14 months versus $\geq 15$ months of age, since passive immunity against measles is likely to persist shortly beyond 12 months., ${ }^{4} 17$ 
Over time, vaccine efficacy against measles, mumps, and/or rubella may decline for some people due to waning immunity. ${ }^{1-4}$ For instance, rubella immunity has been shown to decline after only 12 years, regardless of receiving the second MMR dose at ages four to six or ages nine to $11 .{ }^{19}$ Specifically, $1 \%$ of children $(n=307)$ remained seronegative at one month after the second MMR vaccine was administered as opposed to $10 \%$ of children who were seronegative 12 years later. In addition, measles immunity persists in approximately $80 \%$ of adults immunized as a child. ${ }^{8}$ However, the remaining $20 \%$, including women of child-bearing age, are at risk for measles exposure and the associated consequences. Hence, the MMR vaccine is not foolproof for all pregnant patients due to primary and secondary vaccine failure.

\section{Strategies to improve MMR vaccination among non-immune females}

Improvements of MMR vaccine strategies among females of child-bearing ages are needed to prevent development of CRS, especially for foreign-born females from countries without national immunization schedules. ${ }^{3,20}$ Some of these strategies could include offering the MMR vaccine to eligible, non-vaccinated females at family planning and sexually transmitted disease clinics, at the workplace (e.g., hospital staff, military personnel, and teachers), and at college/university campuses. Furthermore, vaccine advocacy and education are important to expose vaccine truths from myths and to explain the consequences of not vaccinating, emphasizing the harmful and sometimes life-threatening fetal effects after maternal exposure to rubella, along with measles and mumps.

\section{Acknowledgments}

This work was supported by National Institute of Health grants R01 AI33144-15, R01 AI48973-11, and UL1 RR024150-01.

\section{References}

1. Centers for Disease Control and Prevention (CDC). Measles.. In: Atkinson, W.; Wolfe, S.; Hamborsky, J., editors. Epidemiology and Prevention of Vaccine-Preventable Diseases. 12th ed.. Public Health Foundation; Washington, DC: 2011. p. 173-192.

2. Center for Disease Control and Prevention (CDC). Mumps.. In: Atkinson, W.; Wolfe, S.; Hamborsky, J., editors. Epidemiology and Prevention of Vaccine-Preventable Diseases. 12th ed.. Public Health Foundation; Washington, DC: 2011. p. 205-214.

3. Center for Disease Control and Prevention (CDC). Rubella.. In: Atkinson, W.; Wolfe, S.; Hamborsky, J., editors. Epidemiology and Prevention of Vaccine-Preventable Diseases. 12th ed.. Public Health Foundation; Washington, DC: 2011. p. 275-290.

4. Watson JC, Hadler SC, Dykewicz CA, et al. Measles, mumps, and rubella--vaccine use and strategies for elimination of measles, rubella, and congenital rubella syndrome and control of mumps: recommendations of the Advisory Committee on Immunization Practices (ACIP). MMWR Recomm Rep. 1998; 47:1-57. [PubMed: 9639369]

5. Gershon, AA. Chickenpox, measles, and mumps.. In: Remington, JS.; Klein, JO.; Wilson, CB., et al., editors. Infectious Diseases of the Fetus and Newborn Infant. 7th ed.. Elsevier; Philadelphia: 2011. p. 661-705.

6. Plotkin, SA.; Reef, SE.; Cooper, LZ., et al. Rubella.. In: Remington, JS.; Klein, JO.; Wilson, CB., et al., editors. Infectious Diseases of the Fetus and Newborn Infant. 7th ed.. Elsevier; Philadelphia: 2011. p. 861-898.

7. American Academy of Pediatrics (AAP) Committee on Fetus and Newborn and the American College of Obstetrics and Gynecology (ACOG) Committee on Obstetric Practice. Guide to Perinatal Care. 6th ed.. Washington, DC: 2007. Perinatal Infections.; p. 303-348. 
8. Ornoy A, Tenenbaum A. Pregnancy outcome following infections by coxsackie, echo, measles, mumps, hepatitis, polio and encephalitis viruses. Reprod Toxicol. 2006; 21:446-457. [PubMed: 16480851]

9. Eberhart-Phillips JE, Frederick PD, Baron RC, et al. Measles in pregnancy: a descriptive study of 58 cases. Obstet Gynecol. 1993; 82:797-801. [PubMed: 8414327]

10. Centers for Disease Control and Prevention (CDC). Update: Measles --- United States, January-July 2008. MMWR Morb Mortal Wkly Rep. 2008; 57:893-896. [PubMed: 18716580]

11. Centers for Disease Control and Prevention (CDC). Mumps Outbreak --- New York, New Jersey, Quebec, 2009. MMWR Morb Mortal Wkly Rep. 2009; 58:1270-1274. [PubMed: 19940833]

12. Reef SE, Strebel P, Dabbagh A, et al. Progress toward control of rubella and prevention of congenital rubella syndrome--worldwide, 2009. J Infect Dis. 2011; 204:S24-27. [PubMed: 21666168]

13. Valente P, Sever JL. In utero diagnosis of congenital infections by direct fetal sampling. Isr J Med Sci. 1994; 30:414-420. [PubMed: 8034496]

14. Merck \& Co., Inc.. [November 20, 2011] M-M-R IIß (measles, mumps, and rubella vaccine live) [Merck web site]. http://www.merck.com/product/usa/pi_circulars/m/mmr_ii/mmr_ii_pi.pdf.

15. Feinberg, M. [November 11, 2011] Monovalent vaccines no longer available for measles, mumps, rubella [Merck vaccines web site]. Oct 21. 2009 https://www.merckvaccines.com/monovalentMessage_102109.pdf.

16. National Center for Immunization and Respiratory Diseases. General recommendations on immunization --- recommendations of the Advisory Committee on Immunization Practices (ACIP). MMWR Recomm Rep. 2011; 60:1-64.

17. Centers for Disease Control and Prevention (CDC). Measles Prevention. MMWR Morb Mortal Wkly Rep. 1989; 38:1-18.

18. Center for Disease Control and Prevention (CDC). Update: vaccine side effects, adverse reactions, contraindications, and precautions recommendations of the Advisory Committee on Immunization Practices (ACIP). MMWR Morb Mortal Wkly Rep. 1996; 45:1-35. [PubMed: 8531914]

19. LeBaron CW, Forghani B, Matter L, et al. Persistence of rubella antibodies after 2 doses of measles-mumps-rubella vaccine. J Infect Dis. 2009; 200:888-899. [PubMed: 19659440]

20. Gall SA, Poland GA. A maternal immunization program (MIP): Developing a schedule and platform for routine immunization during pregnancy. Vaccine. 2011; 29:9411-9413. [PubMed: 22115415] 